A\&A 389, 629-640 (2002)

DOI: $10.1051 / 0004-6361: 20020586$

(c) ESO 2002

\title{
Dynamics of the fast solar tachocline
}

\section{Dipolar field}

\author{
E. Forgács-Dajka ${ }^{1}$ and K. Petrovay ${ }^{1,2}$ \\ 1 Eötvös University, Department of Astronomy, Budapest, Pf. 32, 1518 Hungary \\ 2 Instute for Theoretical Physics, University of California, Santa Barbara, CA 93106-4030, USA
}

Received 15 January 2002 / Accepted 16 April 2002

\begin{abstract}
One possible scenario for the origin of the solar tachocline, known as the "fast tachocline", assumes that the turbulent diffusivity exceeds $\eta \gtrsim 10^{9} \mathrm{~cm}^{2} \mathrm{~s}^{-1}$. In this case the dynamics will be governed by the dynamogenerated oscillatory magnetic field on relatively short timescales. Here, for the first time, we present detailed numerical models for the fast solar tachocline with all components of the magnetic field calculated explicitly, assuming axial symmetry and a constant turbulent diffusivity $\eta$ and viscosity $\nu$. We find that a sufficiently strong oscillatory poloidal field with dipolar latitude dependence at the tachocline-convective zone boundary is able to confine the tachocline. Exploring the three-dimensional parameter space defined by the viscosity in the range $\log \nu=9-11$, the magnetic Prandtl number in the range $\operatorname{Pr}_{\mathrm{m}}=0.1-10$, and the meridional flow amplitude $\left(-3\right.$ to $\left.+3 \mathrm{~cm} \mathrm{~s}^{-1}\right)$, we also find that the confining field strength $B_{\text {conf }}$, necessary to reproduce the observed thickness of the tachocline, increases with viscosity $\nu$, with magnetic Prandtl number $\nu / \eta$, and with equatorward

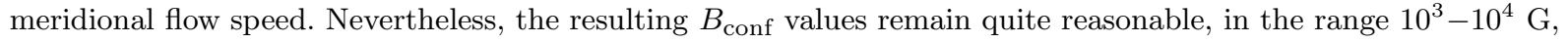
for all parameter combinations considered here. The thickness of the tachocline shows a marked dependence on both time and latitude. The latitude dependence is similar to that inferred by helioseismology, while the time dependence is within the observational errors.
\end{abstract}

Key words. Sun: interior - MHD - Sun: rotation

\section{Introduction}

The tachocline is the thin transitional layer below the solar convective zone between the surface-like differential rotation pervading the convective zone and the rigid rotation of the radiative interior. The existence and properties of this layer have been known from helioseismic studies, recently reviewed by Corbard et al. (2001). The tachocline is known to be extremely thin (Corbard et al. 1998, 1999; Charbonneau et al. 1999). Precise values for its thickness depend on the particular fitting profiles used in helioseismic forward modelling for the residual rotation rate $\Omega-\Omega_{0}$ (where $\Omega_{0}$ is the rotation rate of the solar interior). The scale height of $\Omega-\Omega_{0}$ is generally found to be crudely $0.01-0.02 R_{\odot}$, so that the residual rotation is reduced by one order of magnitude within a layer of barely $\sim 30 \mathrm{Mm}$. Near the equator this layer lies just below the adiabatically stratified convective zone. Inversions seem to suggest

Send offprint requests to: E. Forgács-Dajka, e-mail: E.Forgacs-Dajka@astro.elte.hu that at higher latitudes it is situated at a slightly but significantly higher level, and is apparently also thicker, so that it partly overlaps with the convective zone (Basu \& Antia 2001).

The extreme thinness of the tachocline implies a strongly anisotropic (predominantly horizontal) transport of angular momentum. Several different mechanisms have been proposed for this transport, but it is now widely believed that the magnetic field is instrumental in its origin. Magnetic fields can lead to the required anisotropic transport either directly, via the action of Maxwell stresses, or indirectly, by rendering the rotation profile unstable, and thus giving rise to anisotropic turbulent transport (Gilman \& Dikpati 2000; Gilman 2000). (In contrast, purely hydrodynamic mechanisms are apparently unable to provide the necessary lateral transport - cf. Charbonneau \& MacGregor 1997; Garaud 2001a.)

Depending on the value of the magnetic diffusivity, this magnetic field may either be a weak permanent, primordial field pervading the solar interior, or the strong 
oscillatory field generated by the solar dynamo. A magnetic field oscillating with a circular frequency $\omega_{\text {сус }}=$ $2 \pi / P, P=22$ years is known to penetrate a conductive medium only down to a skin depth of

$H_{\text {skin }}=\left(2 \eta / \omega_{\text {cyc }}\right)^{1 / 2}$

where $\eta$ is the magnetic diffusivity. Comparing this with the tachocline scale height quoted above, it follows that for $\eta \lesssim 10^{8} \mathrm{~cm}^{2} \mathrm{~s}^{-1}$ the dynamo field cannot penetrate the tachocline, and we can expect the tachocline to be pervaded by the internal primordial field. On the other hand, for $\eta \gtrsim 10^{9} \mathrm{~cm}^{2} \mathrm{~s}^{-1}$ the tachocline dynamics should be governed by the dynamo field. As the associated diffusive and Lorentz timescales are also very different, these two cases basically correspond to the case of "slow" and "fast" tachocline, discussed in the literature (see esp. Table I in Gilman 2000; Brun 2001).

The case of a slow tachocline (Gough \& McIntyre 1998) has been studied extensively in recent years by a number of authors. In models with no meridional flow (MacGregor \& Charbonneau 1999; Rüdiger \& Kitchatinov 1997) it was found that for purely molecular diffusivities even a rather weak prescribed internal poloidal field of $10^{-3} \mathrm{G}$ is sufficient to confine the tachocline to its observed thickness if the internal field is fully contained within the radiative zone. The steady toroidal field resulting from the winding up of poloidal field lines may reach kilogauss values. The containment of the internal field is, however, not so easy to explain, given that the surrounding convective zone, with its high diffusivity, is far from being a perfect conductor. On the other hand, for an internal field not contained within the radiative zone Ferraro's law of isorotation predicts that the differential rotation will penetrate deep into the radiative zone. This was indeed confirmed in the numerical models. Recently, Garaud (2001b) performed calculations taking into account the meridional flow and the self-consistent evolution of the poloidal field (with otherwise simplified physics). Her models show that there exists an intermediate range of field strengths where the interaction with the meridional flow indeed leads to the poloidal field being mostly confined to the radiative zone, with a resulting rotation profile that is comparable to the observations. At any rate, these calculations stress the importance of including meridional flows and a self-consistent treatment of the poloidal field.

The alternative case of a fast tachocline, in contrast, has received much less attention. This is so despite that several authors, (e.g. Gilman 2000) noted that the tachocline is more likely to be dynamically coupled to the convective zone than to the radiative zone, and that a part of the tachocline is likely to overlap with the strongly turbulent convective zone, especially at higher latitudes. Faced with this situation, in a recent paper (Forgács-Dajka \& Petrovay 2001, hereafter FDP01) we considered the case of a turbulent tachocline with a turbulent diffusivity of about $\eta=10^{10} \mathrm{~cm}^{2} \mathrm{~s}^{-1}$, pervaded by an oscillatory dipole field. This model can be regarded as the analogue of the models of Rüdiger \& Kitchatinov (1997) and MacGregor \& Charbonneau (1999) for a fast tachocline. It was found that for a sufficiently high value of the field strength, Maxwell stresses in the oscillatory field transported angular momentum efficiently enough to confine the tachocline to its observed thickness. The poloidal field strength necessary for this confinement could also be estimated from a simple analytic relation; with the diffusivity quoted above its value was $2400 \mathrm{G}$.

The analysis of FDP01 was of limited scope, as several simplifying assumptions were made. Only the case of magnetic Prandtl number $\operatorname{Pr}_{\mathrm{m}}=\nu / \eta=1$ ( $\nu$ is the viscosity) was considered, meridional circulation was neglected, the poloidal field was prescribed throughout the computational domain, the applied field was an oscillating dipole instead of a dynamo wave, the diffusivities were arbitrarily prescribed as constant or known functions of radius etc. The aim of the present series of papers is to systematically generalize the model of the fast tachocline by incorporating more and more of the effects neglected in FDP01. As a first step, in the present paper we will incorporate the meridional flow and include a self-consistent calculation of the poloidal field, the importance of which was underlined by the results of Garaud (2001b) in the context of the slow tachocline. We also explore the parameter space a bit more widely by varying the magnetic Prandtl number and the diffusivity.

After formulating the mathematical problem in Sect. 2, results of the model calculations will be presented in Sect. 3. Finally, the Conclusion summarizes the main results.

\section{The model}

\subsection{Equations}

The time evolution of the velocity field $\boldsymbol{v}$ and magnetic field $\boldsymbol{B}$ are governed by the Navier-Stokes and induction equations. We write these in a frame rotating with the fixed internal rotation rate $\Omega_{0}$ :

$$
\begin{aligned}
\partial_{t} \boldsymbol{v} & +(\boldsymbol{v} \cdot \nabla) \boldsymbol{v}=\nabla\left(\frac{1}{2}\left|\boldsymbol{\Omega}_{0} \times \boldsymbol{r}\right|^{2}-V\right)-2\left(\boldsymbol{\Omega}_{0} \times \boldsymbol{v}\right) \\
& -\frac{1}{\rho} \nabla\left(p+\frac{B^{2}}{8 \pi}\right)+\frac{1}{4 \pi \rho}(\boldsymbol{B} \cdot \nabla) \boldsymbol{B}+\frac{1}{\rho} \nabla \cdot \hat{\tau}
\end{aligned}
$$

and

$\partial_{t} \boldsymbol{B}=\nabla \times(\boldsymbol{v} \times \boldsymbol{B})-\nabla \times(\eta \nabla \times \boldsymbol{B})$,

where $\boldsymbol{r}$ is the radius vector, $\hat{\tau}$ is the viscous stress tensor, $\eta$ is the (turbulent) magnetic diffusivity, $V$ is the gravitational potential and $p$ is pressure. These equations are supplemented by the constraints of mass and magnetic flux conservation

$\nabla \cdot(\rho \boldsymbol{v})=0$

$\nabla \cdot \boldsymbol{B}=0$ 
We assume spherical geometry and axisymmetry in all our calculations. The velocity and magnetic fields can be written as

$\boldsymbol{v}=\omega(r, \theta, t) r \sin \theta \boldsymbol{e}_{\phi}+\boldsymbol{v}_{m}$

$\boldsymbol{B}=B(r, \theta, t) \boldsymbol{e}_{\phi}+\nabla \times\left[A(r, \theta, t) \boldsymbol{e}_{\phi}\right]$

where $B(r, \theta, t)$ and $A(r, \theta, t)$ represent the toroidal components of the magnetic field and of the vector potential, respectively; $\omega(r, \theta, t) \equiv \Omega-\Omega_{0}$ is the residual angular velocity and $\boldsymbol{v}_{m}=v_{r} \boldsymbol{e}_{r}+v_{\theta} \boldsymbol{e}_{\theta}$ is the meridional circulation.

As a self-consistent calculation of the meridional circulation throughout the dynamically coupled regions of the convective zone and the tachocline is beyond the scope of this work, we will regard the meridional circulation as given. As a result of the anelastic approximation, the circulation in the spherical shell may be represented by a stream function $\Psi$ so that the components of the meridional circulation can be written as

$$
\begin{aligned}
& v_{r}=\frac{1}{\rho r^{2} \sin \theta} \partial_{\theta} \Psi \\
& v_{\theta}=\frac{-1}{\rho r \sin \theta} \partial_{r} \Psi .
\end{aligned}
$$

In order to present more transparent equations we write the stream function in the following form:

$\Psi=\psi(r) \sin ^{2} \theta \cos \theta$.

the form of the given function $\psi(r)$ specifying the flow.

The evolution of the poloidal magnetic field is, in contrast, self-consistently calculated by integrating Eq. (2) as usual:

$\partial_{t} A+\frac{1}{r \sin \theta}\left(\boldsymbol{v}_{m} \cdot \nabla\right)(r \sin \theta A)=\eta\left(\nabla^{2}-\frac{1}{r \sin \theta}\right) A$.

The components of the viscous stress tensor appearing in the azimuthal component of the Navier-Stokes equation are

$\tau_{\theta \phi}=\tau_{\phi \theta}=\rho \nu \frac{\sin \theta}{r} \partial_{\theta}\left(\frac{v_{\phi}}{\sin \theta}\right)$

$\tau_{\phi r}=\tau_{r \phi}=\rho \nu r \partial_{r}\left(\frac{v_{\phi}}{r}\right)$,

where $\nu$ is the (turbulent) viscosity. With this, the azimuthal components of Eqs. (1-2), including the effects of diffusion, Coriolis force, meridional circulation, toroidal field production by shear, and Lorentz force, read

$$
\begin{aligned}
\partial_{t} \omega= & \left(\partial_{r} \nu+4 \frac{\nu}{r}+\nu \frac{\partial_{r} \rho}{\rho}\right) \partial_{r} \omega+\frac{3 \nu \cot \theta}{r^{2}} \partial_{\theta} \omega \\
& +\nu \partial_{r}^{2} \omega+\frac{\nu}{r^{2}} \partial_{\theta}^{2} \omega+L+M_{1}+C \\
L= & \frac{B}{4 \pi \rho r^{2} \sin \theta}\left(\frac{\partial_{\theta} A}{r}-\cot \theta \partial_{r} A\right) \\
+ & \frac{\partial_{r} B}{4 \pi \rho r^{2} \sin \theta}\left(\partial_{\theta} A-\cot \theta A\right) \\
- & \frac{\partial_{\theta} B}{4 \pi \rho r^{2} \sin \theta}\left(\frac{A}{r}+\partial_{r} A\right)
\end{aligned}
$$

$$
\begin{aligned}
M_{1}= & \frac{1}{r^{2} \rho}\left(2 \cos ^{2} \theta \partial_{r} \psi+\frac{1-3 \cos ^{2} \theta}{r} 2 \psi\right) \omega \\
& +\frac{1-3 \cos ^{2} \theta}{r^{2} \rho} \psi \partial_{r} \omega+\frac{\sin \theta \cos \theta}{r^{2} \rho} \partial_{r} \psi \partial_{\theta} \omega \\
C= & \frac{2 \Omega_{0}}{r^{3} \rho}\left(1-3 \cos ^{2} \theta\right) \psi+\frac{2 \Omega_{0} \cos ^{2} \theta}{r^{2} \rho} \partial_{r} \psi
\end{aligned}
$$

$$
\begin{aligned}
\partial_{t} B= & \left(\frac{\partial_{r} \eta}{r}-\frac{\eta}{r^{2} \sin ^{2} \theta}\right) B+\left(\frac{2 \eta}{r}+\partial_{r} \eta\right) \partial_{r} B \\
& +\frac{\eta}{r^{2}} \cot \theta \partial_{\theta} B+\eta \partial_{r}^{2} B+\frac{\eta}{r^{2}} \partial_{\theta}^{2} B+M_{2} \\
M_{2}= & \frac{\psi}{r^{2} \rho}\left[\left(3 \cos ^{2} \theta-1\right)\left(\frac{1}{r}+\frac{\partial_{r} \rho}{\rho}\right)\right] B \\
& -\frac{\partial_{r} \psi}{r^{2} \rho} \cos ^{2} \theta B+\frac{\psi}{r^{2} \rho}\left(1-3 \cos ^{2} \theta\right) \partial_{r} B
\end{aligned}
$$

while Eq. (10) can be written as

$$
\begin{aligned}
\partial_{t} A & =\frac{-\eta}{r^{2} \sin ^{2} \theta} A+\frac{2 \eta}{r} \partial_{r} A+\frac{\eta \cot \theta}{r^{2}} \partial_{\theta} A \\
& +\eta \partial_{r}^{2} A+\frac{\eta}{r^{2}} \partial_{\theta}^{2} A+M_{3} \\
M_{3} & =\frac{1}{r^{2} \rho}\left(\frac{1-3 \cos ^{2} \theta}{r} \psi+\cos ^{2} \theta \partial_{r} \psi\right) A \\
& +\frac{\psi}{r^{2} \rho}\left(1-3 \cos ^{2} \theta\right) \partial_{r} A+\frac{\sin \theta \cos \theta}{r^{2} \rho} \partial_{r} \psi \partial_{\theta} A .
\end{aligned}
$$

In these formulae $L$ denotes the terms associated with the Lorentz force, $C$ denotes the terms associated with the Coriolis force and $M_{1}, M_{2}, M_{3}$ denotes the terms associated with the advection by meridional circulation.

\subsection{Boundary and initial conditions}

The computational domain for the present calculations consists of just the upper part of the radiative interior, between radii $r_{\mathrm{in}}$ and $r_{\mathrm{bcz}}$, where $r_{\mathrm{bcz}}$ is the radius of the bottom of the convection zone. For the integration of Eqs. (13-15) we use the following boundary conditions.

At the pole and the equator axial and equatorial symmetry is required for $\omega$, and dipole symmetry for the magnetic field:

$$
\begin{array}{ll}
\partial_{\theta} \omega=B=A=0 & \text { at } \theta=0, \\
\partial_{\theta} \omega=B=\partial_{\theta} A=0 & \text { at } \theta=\pi / 2 .
\end{array}
$$

The bottom of our box is supposed to be a rigidly rotating perfect conductor - a good approximation for the solar interior. The rigid rotation speed is fixed at the observed rate $\Omega_{0} / 2 \pi=437 \mathrm{nHz}$. Thus, the lower boundary conditions are:

$\omega=B=A=0 \quad$ at $r=r_{\text {in }}$.

Note that the fixed internal rotation rate $\Omega_{0}$ is in general not equal to the rate at which the interior of our models would rotate if the lower rotation rate were not prescribed. (In other words, the fixed rate does not in general ensure zero net torque at the lower boundary.) This leads 
to a spurious radial differential rotation in the radiative zone below the tachocline. In models where this effect is strong, it is a sign that the model does not represent well the observations as it would spontaneously lead to an internal rotation rate that differs significantly from what is observed. As, however, the magnetic field geometry considered here is still not realistic, firm conclusions on this would be premature anyway.

The lower boundary needs also to be placed sufficiently deep in the solar interior to make sure that the confinement of the tachocline is not an artefact caused by the rigid rotation imposed at the bottom. For this reason, in most calculations presented here we set $r_{\text {in }}=0.4 R_{\odot}$, even though only the upper part of our computational domain is shown in many of the figures.

At the upper boundary of our domain, i.e. at the bottom of the convective zone, we use the following boundary conditions:

$\omega=\Omega_{\mathrm{bcz}}-\Omega_{0}$

$A=A_{0} \sin \theta \cos \left(\omega_{\mathrm{cyc}} t\right) \quad$ at $r=r_{\mathrm{bcz}}$,

$B=0$.

The physical assumptions behind these conditions are the following. For $\omega$ we suppose that the rotation rate can be described with the same expression as in the upper part of the convection zone. In accordance with the observations of the GONG network, the expression used for $\Omega_{\mathrm{bcz}}$ is

$\frac{\Omega_{\mathrm{bcz}}}{2 \pi}=456-72 \cos ^{2} \theta-42 \cos ^{4} \theta \mathrm{nHz}$.

Our upper boundary condition on the poloidal field is the simplest possible representation of the poloidal component of the solar dynamo field, a dipole oscillating with a period of 22 years. The factor $A_{0}$ fixes the poloidal field amplitude. Finally, the upper boundary condition chosen for the toroidal field assumes that the toroidal field is negligible in the convective zone compared to its value in the tachocline. Such a hypothetical situation is in line with current thinking in dynamo theory, and it may be a natural consequence of buoyancy-driven instabilities effectively removing any toroidal flux from the convective zone.

The initial conditions chosen for all calculations are

$$
\begin{array}{ll}
\omega(r, \theta, t=0)=\Omega_{\mathrm{bcz}}-\Omega_{0} & \text { at } r=r_{\mathrm{bcz}} \\
\omega(r, \theta, t=0)=0 & \text { at } r<r_{\mathrm{bcz}} \\
B(r, \theta, t=0) \equiv 0 & \\
A(r, \theta, t=0)=A_{0} \sin \theta & \text { at } r=r_{\mathrm{bcz}} \\
A(r, \theta, t=0)=0 & \text { at } r<r_{\mathrm{bcz}} .
\end{array}
$$

Some test runs with other initial conditions (with significant differential rotation throughout the volume) were also performed. After stationary periodic behaviour sets in, the results were found to be independent of the initial conditions chosen.

\subsection{Numerical method}

We used a time relaxation method with a finite difference scheme first order accurate in time to solve the equations.
A uniformly spaced grid is set up with 128 grid points in the $r$ direction and 32 grid points in the $\theta$ direction.

Our calculations are based on a more recent version of the solar model of Guenther et al. (1992).

Starting from the initial conditions (20), the solution is allowed to evolve in time until it relaxes to a very nearly periodic behaviour. The timescale needed for this is the diffusive timescale

$t_{\mathrm{dif}}=\frac{\left(r_{\mathrm{bcz}}-r_{\mathrm{in}}\right)^{2}}{\operatorname{Min}(\nu, \eta)}$,

where $\operatorname{Min}(\nu, \eta)$ is the lowest value of viscosity or magnetic diffusivity in the domain. Physically we would expect this value to be close to the molecular viscosity. Using this value in the computations would, however, lead to a prohibitively high number of timesteps to relaxation. (Clearly, the runtime of our computations must be chosen to well exceed $t_{\text {dif }}$ to reach relaxation.) Consequently, in the present paper we only consider cases where the diffusivities are constant throughout the domain. Note, however, that in FDP01 a case with the diffusivity significantly reduced towards the interior was also computed, and no qualitative changes in the character of the solution were found.

The question may arise, to what extent does the use of such a high viscosity in the deep radiative zone distort the results? The answer is clearly that the main difference between models with high and low diffusivities is the diffusive timescale over which the model evolves to equilibrium. Once equilibrium is reached, the azimuthal component of the equation of motion reduces to a Laplace equation (neglecting meridional circulation and magnetic fields), so the solution is universal and independent of the diffusivity. The equilibrium solution is thus not expected to be strongly influenced.

On the other hand, for realistically low (such as molecular) diffusivities equilibration takes much longer than the age of the Sun. Thus, an internal differential rotation, induced by solar wind torques or as a pre-MS relict, may not be suppressed. It is therefore important to make it clear (as we already did in FDP01) that the present work does not propose that dynamo-generated fields can explain the overall lack of differential rotation in the deep radiative zone, just that they dominate the dynamics of the tachocline itself. The lack of differential rotation in the deep radiative zone still needs to be explained by an internal magnetic field independent of the dynamo.

\section{Results and discussion}

\subsection{Reference case}

First we present results for a case with $\operatorname{Pr}_{\mathrm{m}}=1, \eta=$ $\nu=10^{10} \mathrm{~cm}^{2} \mathrm{~s}^{-1}$, and no meridional circulation $(\psi=0)$. This "reference case" corresponds to that computed in FDP01, except that the poloidal field is now not prescribed throughout the volume, but explicitly calculated. The results are shown in Fig. 1. 

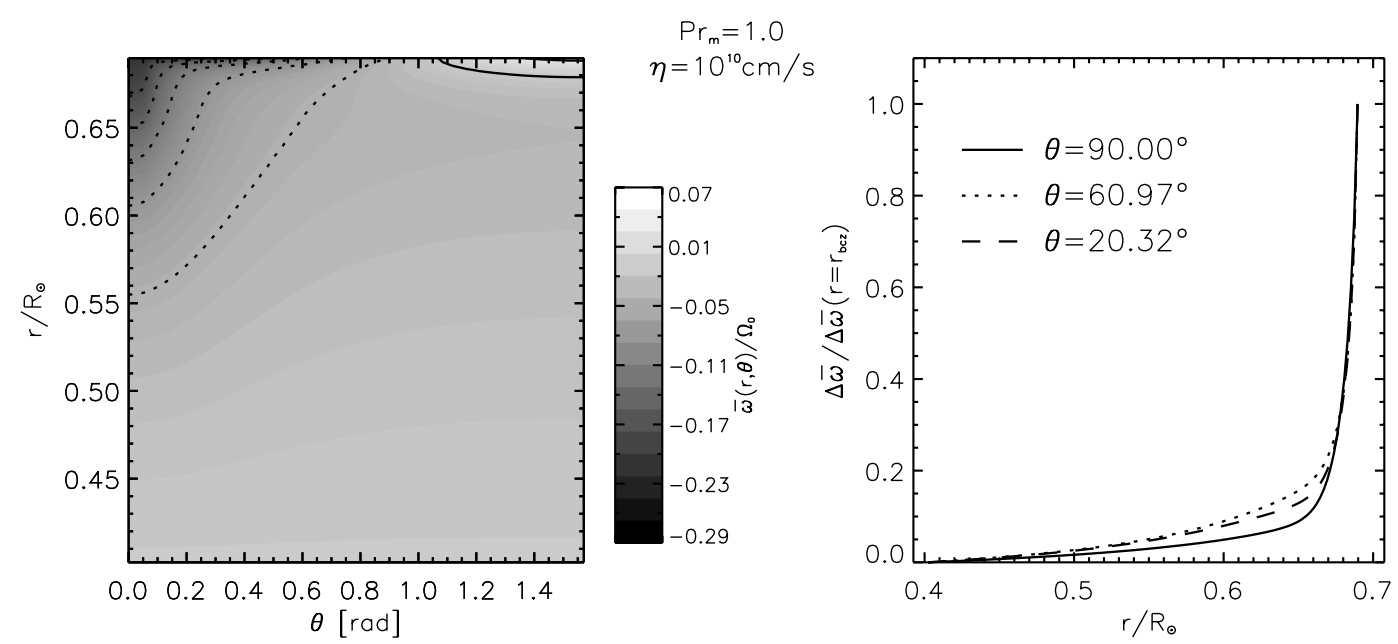

Fig. 1. Spreading of the differential rotation into the radiative interior for $\eta=\nu=10^{10} \mathrm{~cm}^{2} \mathrm{~s}^{-1}$. Left-hand panel: contours of the time-average of the angular rotation rate $\omega(r, \theta, t)$ under one dynamo period. Equidistant contour levels are shown, separated by intervals of $100 \mathrm{nHz} / \Omega_{0}$, starting from 0 towards both non-negative (solid) and negative (dashed) values. Right-hand panel: normalized differential rotation amplitude $\Delta \omega$ at different latitudes as a function of radius. The peak amplitude of the poloidal magnetic field is $B_{\mathrm{p}} \sim 2570 \mathrm{G}$.
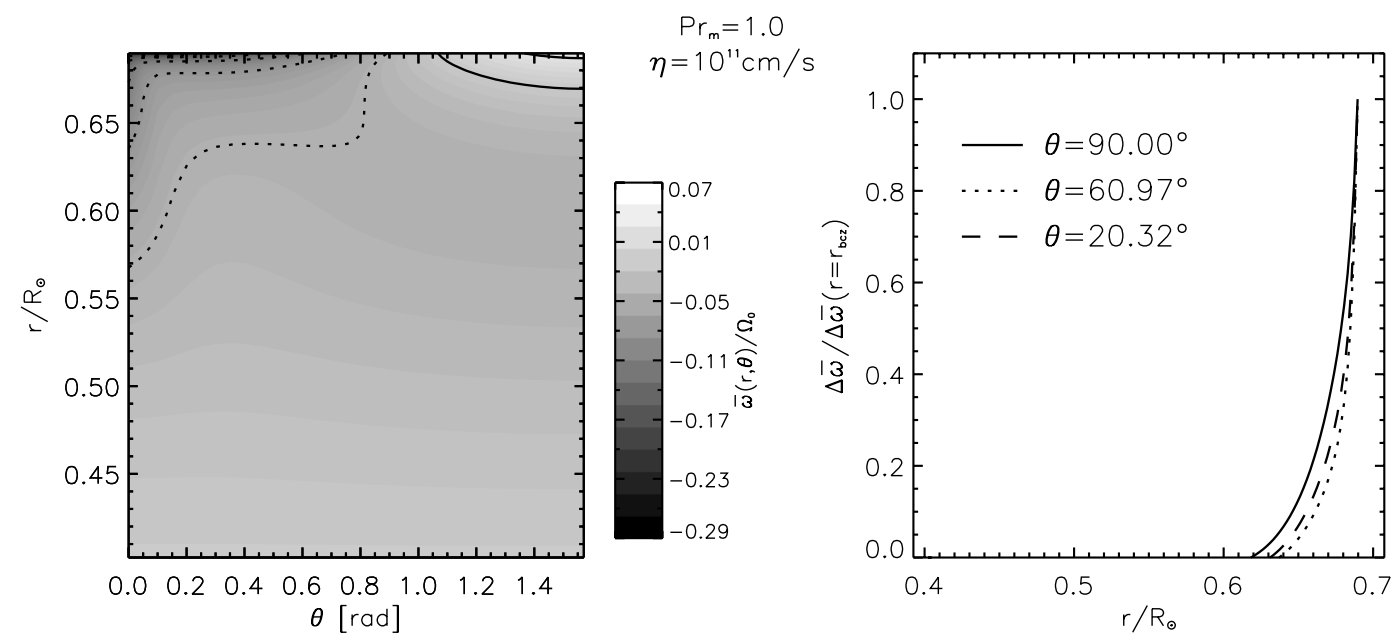

Fig. 2. Same as in Fig. 1 for $\eta=\nu=10^{11} \mathrm{~cm}^{2} \mathrm{~s}^{-1}$. The peak amplitude of the poloidal magnetic field is $B_{\mathrm{p}} \sim 6660 \mathrm{G}$.
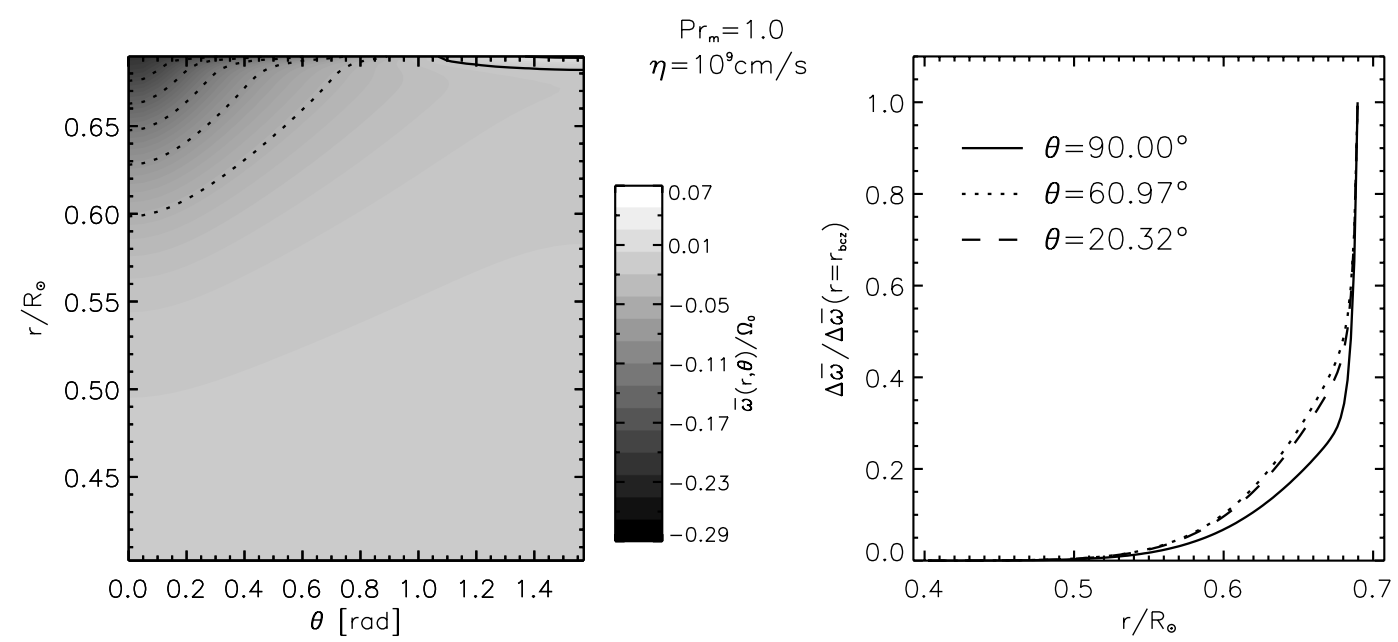

Fig. 3. Same as in Fig. 1 for $\eta=\nu=10^{9} \mathrm{~cm}^{2} \mathrm{~s}^{-1}$. The peak amplitude of the poloidal magnetic field is $B_{\mathrm{p}} \sim 1386 \mathrm{G}$. 
Elapsed time inside of a cycle: 0.00 year $\quad \eta=10^{10} \mathrm{~cm} / \mathrm{s} \quad \operatorname{Pr}_{\mathrm{m}}=1.0$
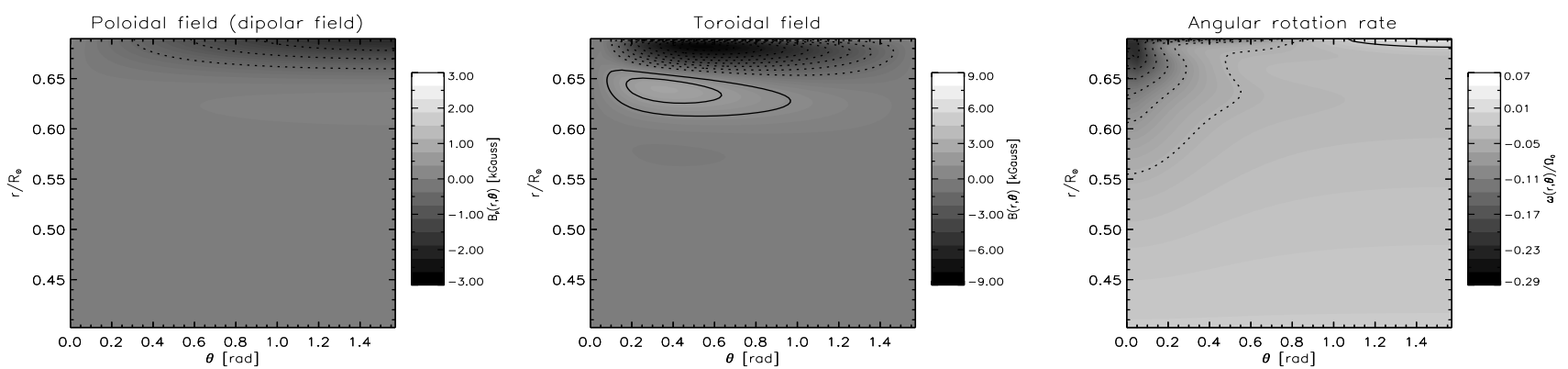

Elapsed time inside of a cycle: 3.34 year $\quad \eta=10^{10} \mathrm{~cm} / \mathrm{s} \quad \operatorname{Pr}_{\mathrm{m}}=1.0$
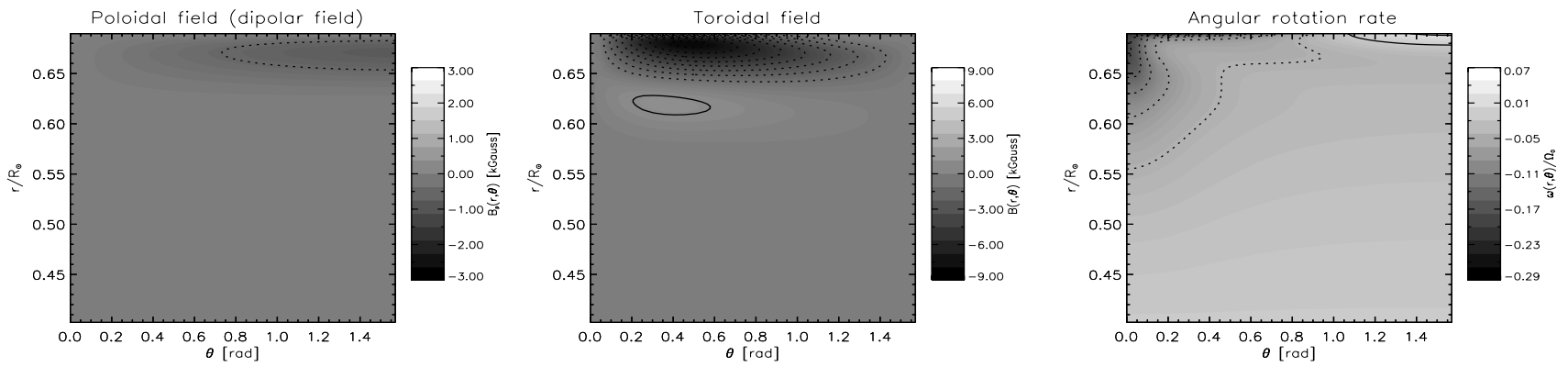

Elapsed time inside of a cycle: 7.79 year
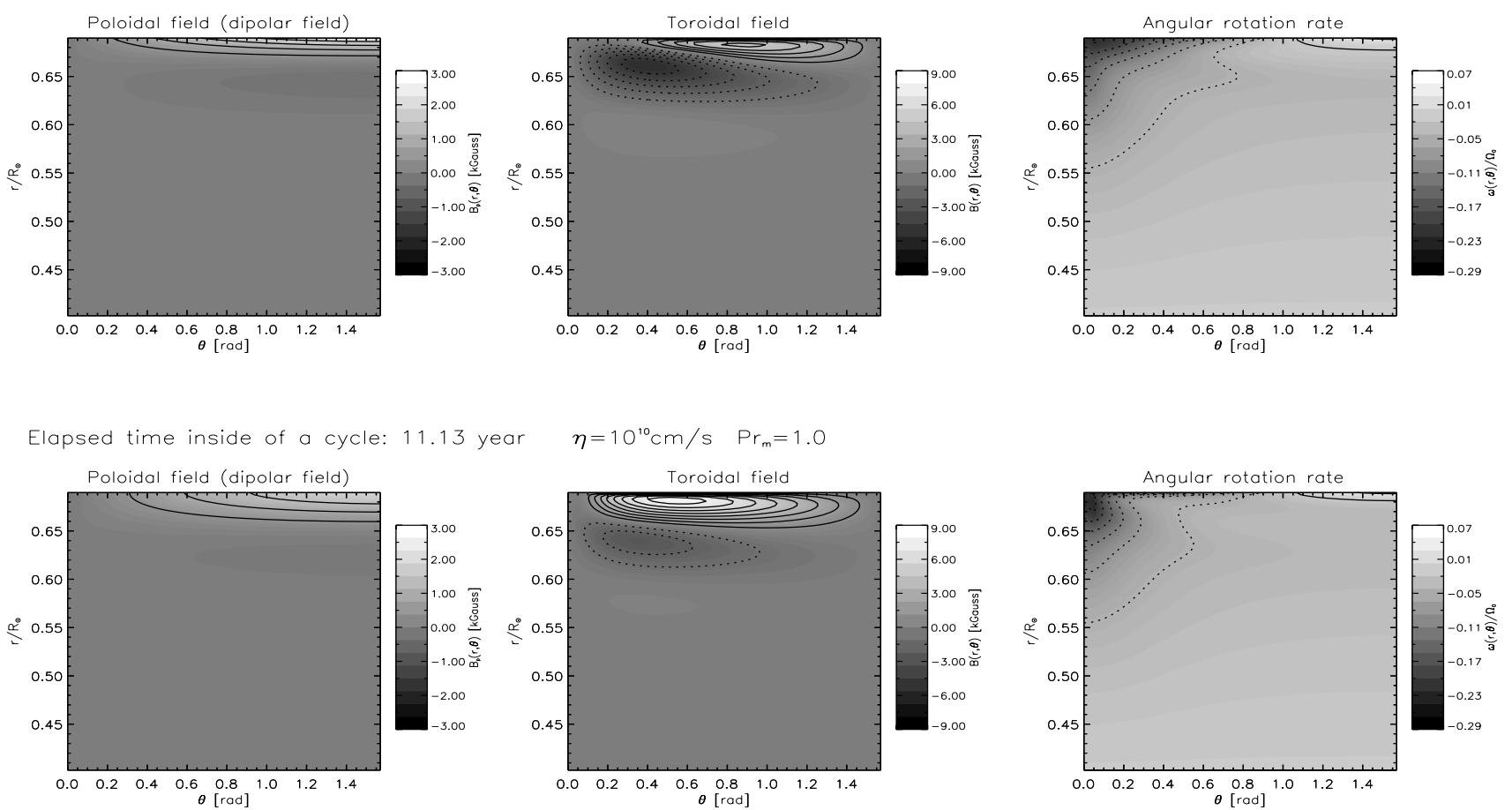

Fig. 4. Snapshots of the solution at four cycle phases for $\eta=\nu=10^{10} \mathrm{~cm}^{2} \mathrm{~s}^{-1}$. Left-hand panels: contours of poloidal magnetic field strength. Equidistant contour levels are shown, separated by intervals of $500 \mathrm{G}$. Middle panels: contours of toroidal magnetic field strength. Equidistant contour levels are shown, separated by intervals of 1000 G. Right-hand panels: contours of angular rotation rate, as in Fig. 1. 
In the left-hand panels we plotted the contours of the time-average of the angular rotation rate, which is defined as

$\bar{\omega}(r, \theta)=\frac{1}{P_{\text {сус }}} \int_{t}^{t+P_{\text {cyc }}} \omega(r, \theta, t) \mathrm{d} t$.

In the right-hand panels the differential rotation amplitude $\Delta \omega$ is defined as

$\Delta \omega=\omega(r, \theta, t)-\int_{0}^{\pi / 2} \omega(r, \theta, t) \sin \theta \mathrm{d} \theta$

$\Delta \bar{\omega}=\bar{\omega}(r, \theta)-\int_{0}^{\pi / 2} \bar{\omega}(r, \theta) \sin \theta \mathrm{d} \theta$

The thickness of the tachocline will be defined throughout this paper as the scale height of $\Delta \omega$ (i.e. the depth from the top of our domain where $\Delta \omega$ is reduced by $1 / \mathrm{e}$ ). By "mean thickness", in turn, we will refer to the scale height of $\Delta \bar{\omega}$.

The results shown in Fig. 1 are in accordance with those in FDP01. In particular, we confirm that an oscillatory poloidal field with dipolar latitude dependence at the tachocline-convective zone boundary is able to confine the thickness of tachocline. The effectivity of this confinement increases (i.e. the thickness of the tachocline decreases) with the field strength.

This conclusion is basically valid for all the cases studied in this paper, though the field strength necessary to just reproduce the observed equatorial mean thickness of the tachocline, to be called the confining field strength $B_{\text {conf }}$, for brevity, varies from case to case, as do the structural details of the resulting tachocline. Though in order to find the right value of $B_{\text {conf }}$ we experimented with many different field strengths for each parameter combination, in this paper we only present results computed with $B=B_{\text {conf }}$ (cf. FDP01 for calculations with other field strengths). The value of the confining field strength is found to be $\sim 2600 \mathrm{G}$ for the reference case, very close to the value found in FDP01.

In what follows, we will systematically study the effects of varying the parameters of the model on the resulting tachocline structure and on the value of the confining field strength. First, we fix the value of the magnetic Prandtl number as $\operatorname{Pr}_{\mathrm{m}}=\nu / \eta=1.0$, while we change the amplitude of the magnetic diffusivity. Second, we examine the influence of varying the magnetic Prandtl number on the radial spreading of the differential rotation into the radiative interior. In these two cases we ignore the meridional flow. Finally, we will focus on the effect of introducing a meridional flow.

Computer animations illustrating the time development of some of our solutions can be downloaded from the following web site:

http://astro.elte.hu/kutat/sol/fast1/fast1e.html

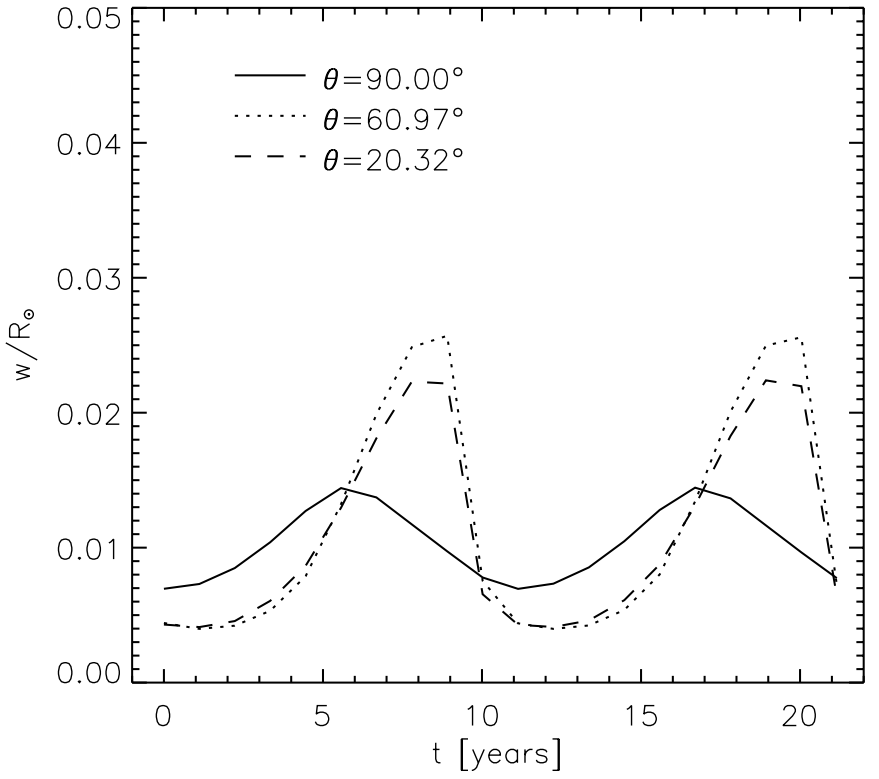

Fig. 5. The thickness of the tachocline at different latitudes as a function of time for the case in Fig. 1.

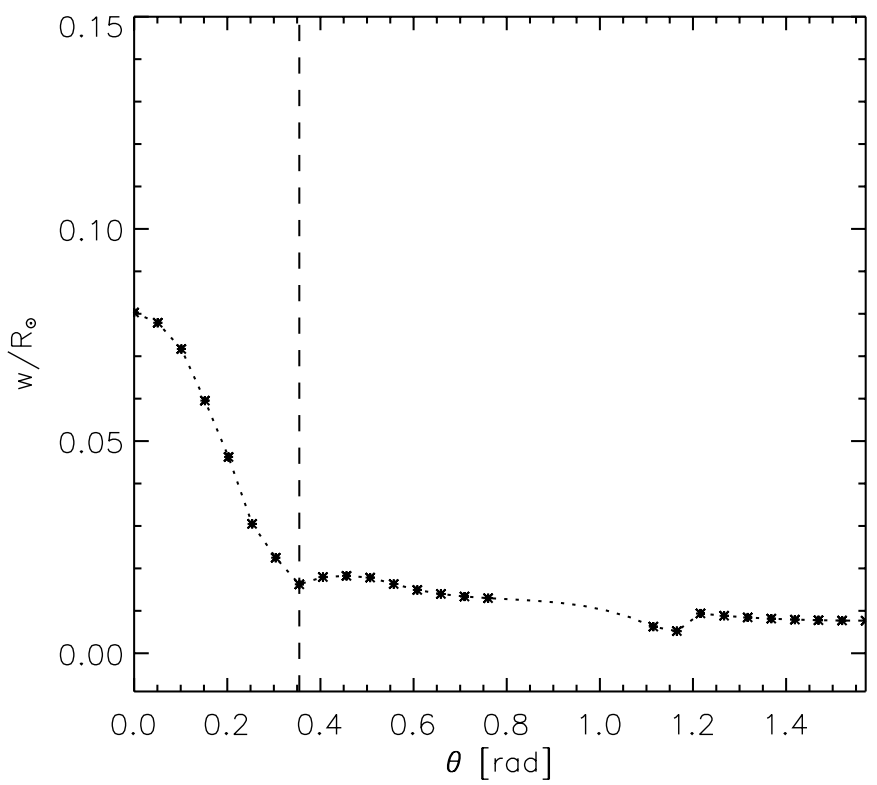

Fig. 6. Latitudinal variation of the mean tachocline thickness for the case in Fig. 1. A vertical dashed line indicates the poleward limit of reliable helioseismic data.

\subsection{Varying the diffusivity}

In this subsection, the value of the magnetic Prandtl number was fixed, while we studied the effects of varying the diffusivity. The results are shown in Figs. 1-3.

In line with the analytic estimates of FDP01, we find that $B_{\text {conf }}$ increases with the value of the diffusivity. Given that the equipartition field strength in the deep convective zone is $\sim 10^{4} \mathrm{G}$, the value of $B_{\text {conf }}$ is quite reasonable even with a diffusivity of $10^{11} \mathrm{~cm}^{2} \mathrm{~s}^{-1}$.

Beside increasing the value of $B_{\text {conf }}$, a higher diffusivity has other effects on the solution, too. The fast tachocline shows a quite marked time- and latitude-dependence in 

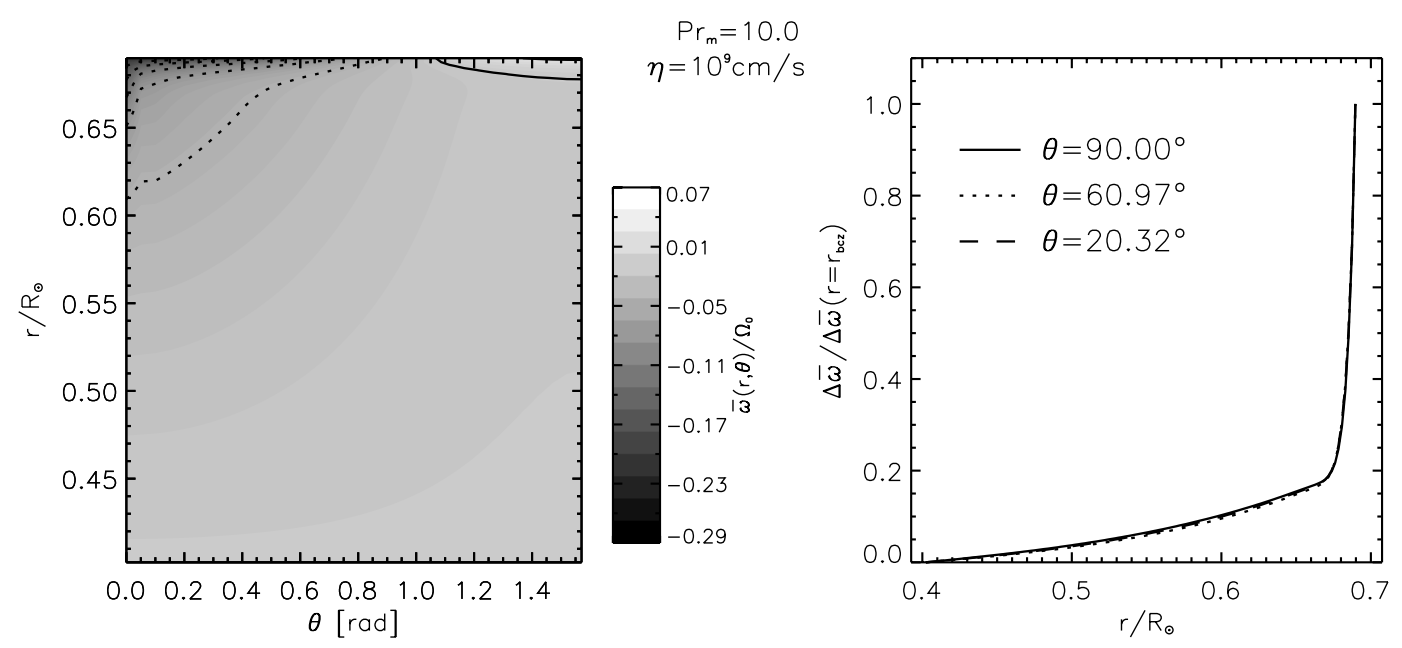

Fig. 7. Same as in Fig. 1 for $\operatorname{Pr}_{\mathrm{m}}=10.0$, where $\eta=10^{9} \mathrm{~cm}^{2} \mathrm{~s}^{-1}$ and $\nu=10^{10} \mathrm{~cm}^{2} \mathrm{~s}^{-1}$. The peak amplitude of the poloidal magnetic field is $B_{\mathrm{p}} \simeq 8531 \mathrm{G}$.
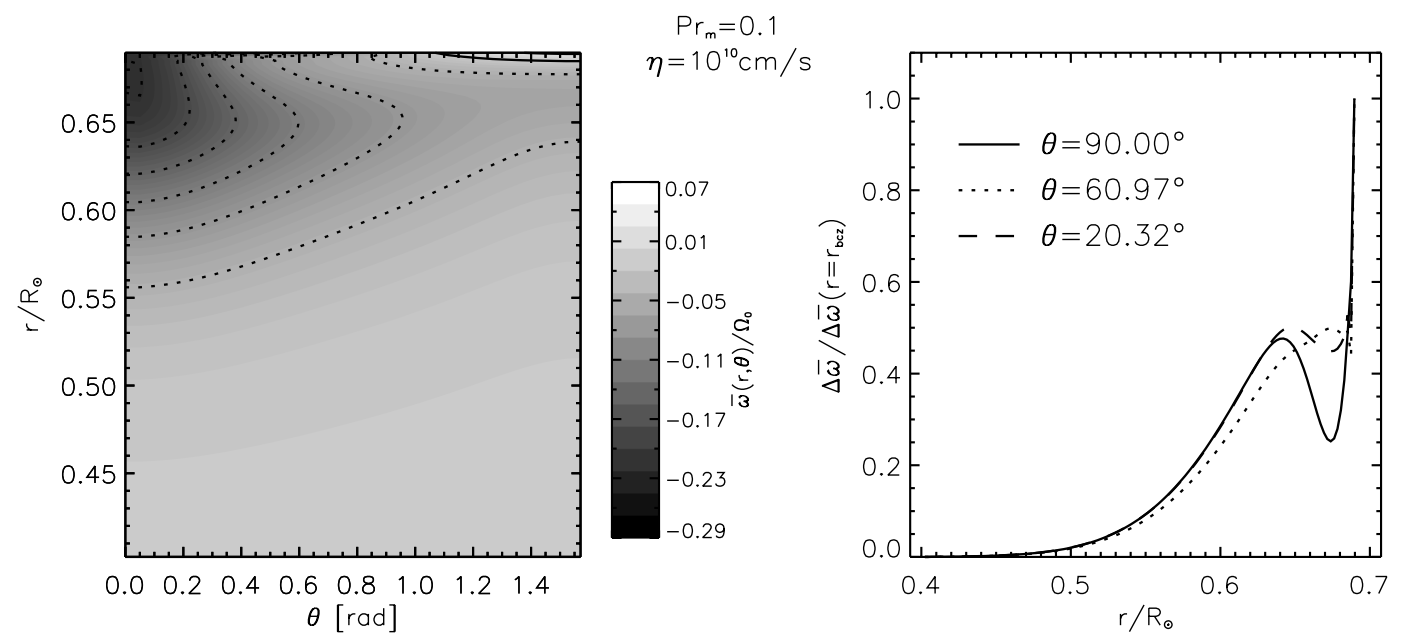

Fig. 8. Same as in Fig. 1 for $\operatorname{Pr}_{\mathrm{m}}=0.1$, where $\eta=10^{10} \mathrm{~cm}^{2} \mathrm{~s}^{-1}$ and $\nu=10^{9} \mathrm{~cm}^{2} \mathrm{~s}^{-1}$. The peak amplitude of the poloidal magnetic field is $B_{\mathrm{p}} \simeq 1028 \mathrm{G}$.

our models, as illustrated in Fig. 4 for the reference case. As a consequence, the thickness of the tachocline depends both on latitude and cycle phase. In particular, the tachocline tends to be significantly wider at higher latitudes on the mean, while it is also more time-dependent near the poles (Fig. 5). While the relative shortness of seismic data sets does not permit firm conclusions about the cycle dependence of tachocline properties yet, the overall form of the mean thickness-latitude relation (Fig. 6) is in good qualitative agreement with the available helioseismic information (cf. Fig. 9 in Basu \& Antia 2001). The amplitude of these variations within the latitude interval where the helioseismic results are reliable $\left(\theta \gtrsim 20^{\circ}\right)$ also seems to be in agreement with the observations.

For a fixed value of the field strength, a higher value of diffusivity tends to reduce the amplitude of the spatiotemporal variations. On the other hand, for higher diffusivities $B_{\text {conf }}$ is also higher, so for this field strength a stronger dynamical variation is imposed on the flow. Nevertheless, at least for the reference case, the time dependence is well within the observational errors as shown e.g. in Fig. 4 of Basu \& Antia (2001).

It must be noted of course that, as our imposed upper boundary condition on the poloidal field, a simple oscillating dipole, may not be very realistic, the detailed spatiotemporal properties of the solution should be regarded with caution. Among such features we may mention the poleward drift of the resulting rotational pattern, apparent e.g. in the phase lag between different latitudes in Fig. 5. (Cf. the same trend in Fig. 10 of FDP01.)

\subsection{Varying the magnetic Prandtl number}

Figures 7 and 8 illustrate the effect of magnetic Prandtl numbers other than 1 on the solution. As expected on the basis of the analytical estimates of FDP01, tachocline confinement is less efficient for high magnetic Prandtl numbers, so that higher values of $B_{\text {conf }}$ are necessary 


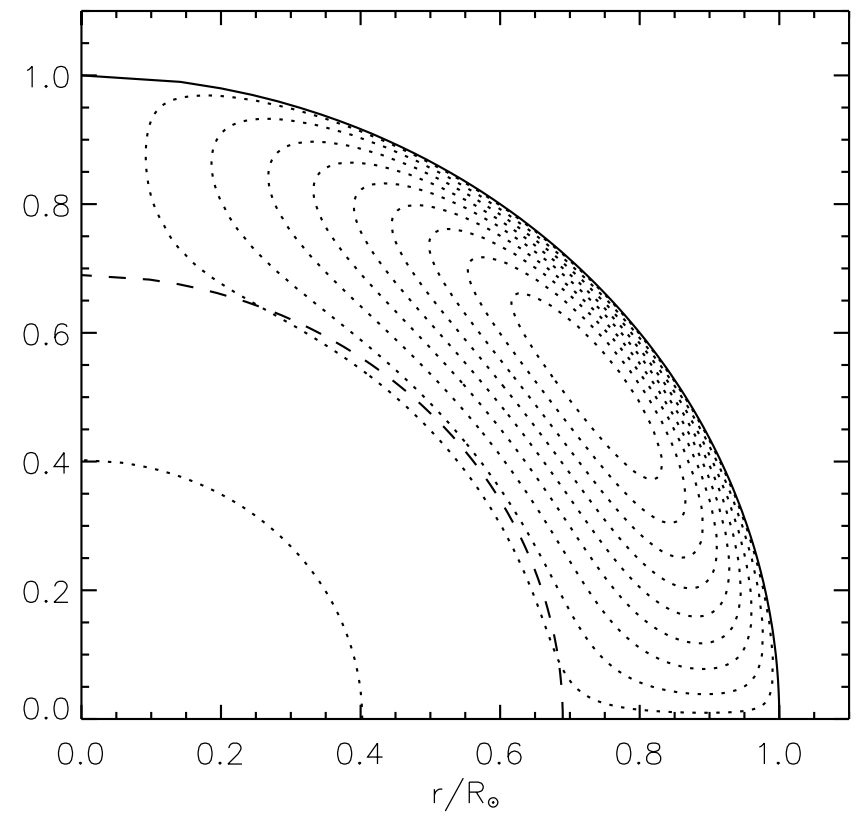

Fig. 9. Streamlines of the meridional circulation prescribed in Eq. (25), with the following parameter values: $\psi_{0}=-10^{20}$, $k=1, r_{0}=\left(R_{\odot}-r_{\mathrm{mc}}\right) / 30$ and $\Gamma=3.4 \times 10^{10}$. Dotted lines represent counterclockwise circulation. The amplitude of the flow is $\sim 3 \mathrm{~cm} \mathrm{~s}^{-1}$ in our computational domain (between the dashed and dotted circles).

if $\operatorname{Pr}_{\mathrm{m}}>1$. On the other hand, a higher magnetic Prandtl number (i.e. a relatively higher viscosity) is in general beneficial for the overall smoothness of the solution, leading to less strong dependence of $\omega$ on time and latitude. In the low $\operatorname{Pr}_{\mathrm{m}}$ case, in turn, strong spatiotemporal variations in $\omega$ become prevalent, leading to a complex nonmonotonic behaviour of the rotational velocity as a function of radius and latitude.

\subsection{Influence of the meridional flow}

In the case of a fast tachocline dynamically coupled to the convective zone, as assumed here, we expect that any meridional circulation present in the tachocline is unseparable from the complex problem of meridional circulation in the convective zone. It is far beyond the scope of this paper to attempt to model this circulation in a consistent way. Instead, we will limit ourselves to regard the effects of a meridional circulation with a simple and plausible, prescribed spatial structure, and a realistic amplitude.

It is known from direct and seismic studies that the meridional circulation near the photosphere is poleward, with a peak amplitude of $\sim 20 \mathrm{~m} \mathrm{~s}^{-1}$ (Komm et al. 1993; Latushko 1994). If we assume that the meridional flow in the convective zone+tachocline system follows a simple one-cell pattern, the circulation in the tachocline will be equatorward. The alternative possibility of a two-cell pattern with poleward flow in the tachocline, however, should also be left open for now (Küker \& Stix 2001).

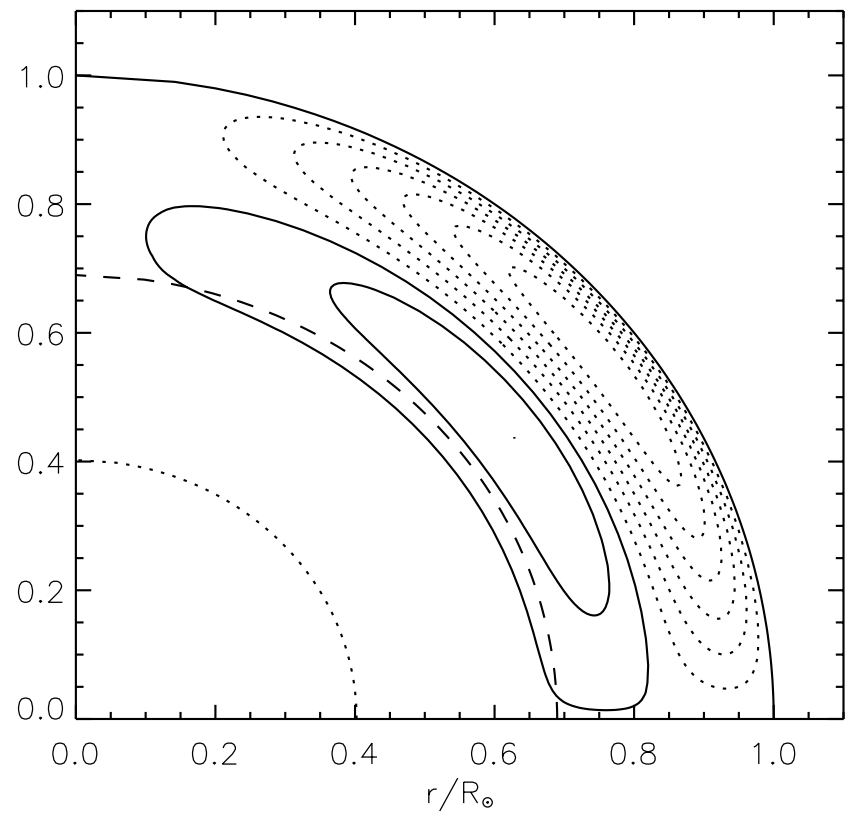

Fig. 10. Same as in Fig. 9 with $\psi_{0}=10^{20}, k=2$. Solid and dotted lines represent clockwise and counterclockwise circulation, respectively.

An expression for the function $\psi(r)$, defined in Eq. (9), that encompasses both possibilities is

$\psi(r)=\psi_{0} \sin \left[\frac{k \pi\left(r-r_{\mathrm{mc}}\right)}{R_{\odot}-r_{\mathrm{mc}}}\right] \exp \left(\frac{\left(r-r_{0}\right)^{2}}{\Gamma^{2}}\right)$,

where $\psi_{0}$ sets the amplitude of the meridional circulation, $r_{0}, \Gamma$ and $k$ are geometric parameters and $r_{\mathrm{mc}}$ is the radius value down to which the meridional flow penetrates from the base of the convective zone. Keeping in mind the observed depth of tachocline, here we set $r_{\mathrm{mc}}=4.6 \times 10^{10} \mathrm{~cm}$.

The strong subadiabatic stratification of the solar interior sets an upper limit to the amplitude of the meridional flow. The timescale of any meridional circulation cannot be shorter than the relevant thermal diffusive timescale (to allow moving fluid elements to get rid of their buoyancy). In the present case the relevant heat conductivity is the turbulent one, so for turbulent Prandtl numbers not very different from unity, the shortest possible timescale for meridional circulation is just comparable to the viscous timescale. An elementary estimate yields $v_{\text {max }} \sim r_{\text {bcz }} / t_{\text {dif }} \sim 10 \mathrm{~cm} \mathrm{~s}^{-1}$.

The flow parameters used in our calculations were chosen to produce simple smooth one- and two-celled flow patterns obeying the amplitude constraint discussed above, while reproducing the observed flow speed near the surface. The flow patterns are shown in Figs. 9 and 10. In these cases, the speed of the horizontal motion is $\sim 3 \mathrm{~cm} \mathrm{~s}^{-1}$ in the upper part of the radiative interior.

The results with the meridional circulation included can be seen in Figs. 11 and 12. The poloidal field amplitude used here was the same as in the reference case. It is apparent that an equatorward flow in the tachocline region reduces somewhat the efficiency of the confinement 

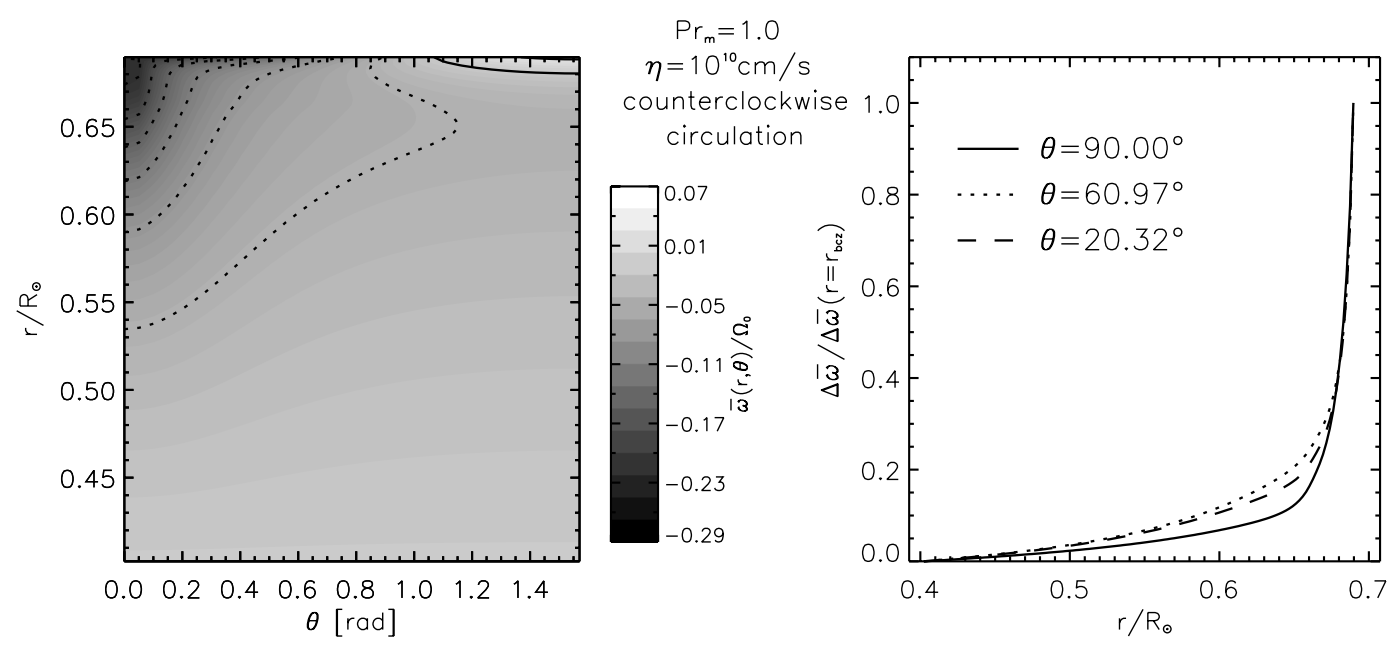

Fig. 11. Same as in Fig. 1, but including the meridional circulation shown in Fig. 9.
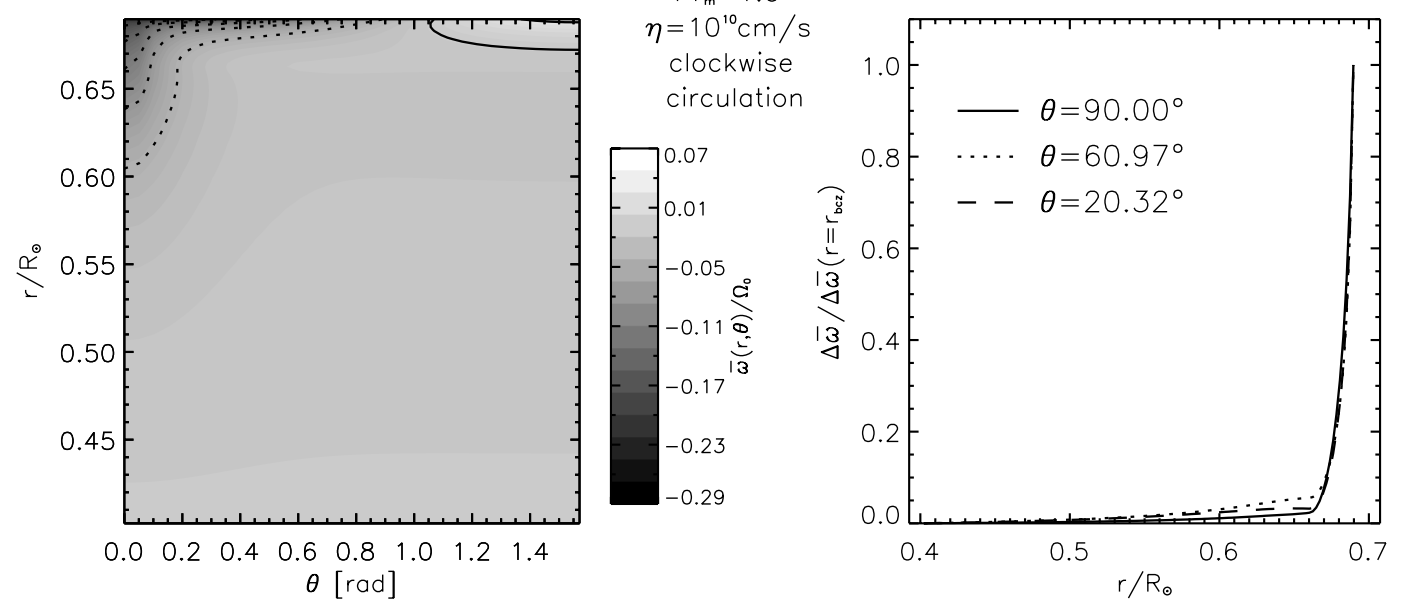

Fig. 12. Same as in Fig. 1, but including the meridional circulation shown in Fig. 10.

(the tachocline becomes slightly thicker), though the effect is not dramatic. A poleward flow, on the other hand, significantly improves the efficiency of the confinement.

In fact, meridional circulation alone, without any imposed magnetic field, can also have a significant effect on the thickness of the tachocline, as it can be seen by comparing Figs. 13 and 14.

The relative importance of meridional circulation scales approximately with the ratio of the diffusive/advective timescales $v / \nu$; thus, for a higher value of $\nu$, a proportionally higher flow speed is needed to reproduce the same tachocline thickness.

\section{Conclusion}

We have begun a systematic study of the dynamics of the fast solar tachocline arising under the assumption that the turbulent diffusivity in the tachocline exceeds $\eta \gtrsim 10^{9} \mathrm{~cm}^{2} \mathrm{~s}^{-1}$. We extended the earlier preliminary analysis of FDP01 by consistently computing the evolution of the poloidal field, and by exploring the threedimensional parameter space defined by the viscosity in the range $\log \nu=9-11$, the magnetic Prandtl number in the range $\operatorname{Pr}_{\mathrm{m}}=0.1-10$, and the meridional flow amplitude $\left(-3\right.$ to $\left.+3 \mathrm{~cm} \mathrm{~s}^{-1}\right)$. The feedback of the Lorentz force to the meridional circulation was neglected.

Confirming the results in FDP01, we found that, for basically all parameter combinations considered, an oscillatory poloidal field with dipolar latitude dependence at the tachocline-convective zone boundary is able to confine the thickness of tachocline. (Note, however, that for low magnetic Prandtl numbers the flow becomes rather complex, and a simple, smooth, monotonic tachocline layer does not form.) The effectivity of this confinement increases (i.e. the thickness of the tachocline decreases) with the field strength. The confining field strength $B_{\text {conf nec- }}$ essary to just reproduce the observed mean equatorial thickness of the tachocline is found to increase with increasing viscosity, magnetic Prandtl number $\nu / \eta$, and meridional flow speed (if considered positive 

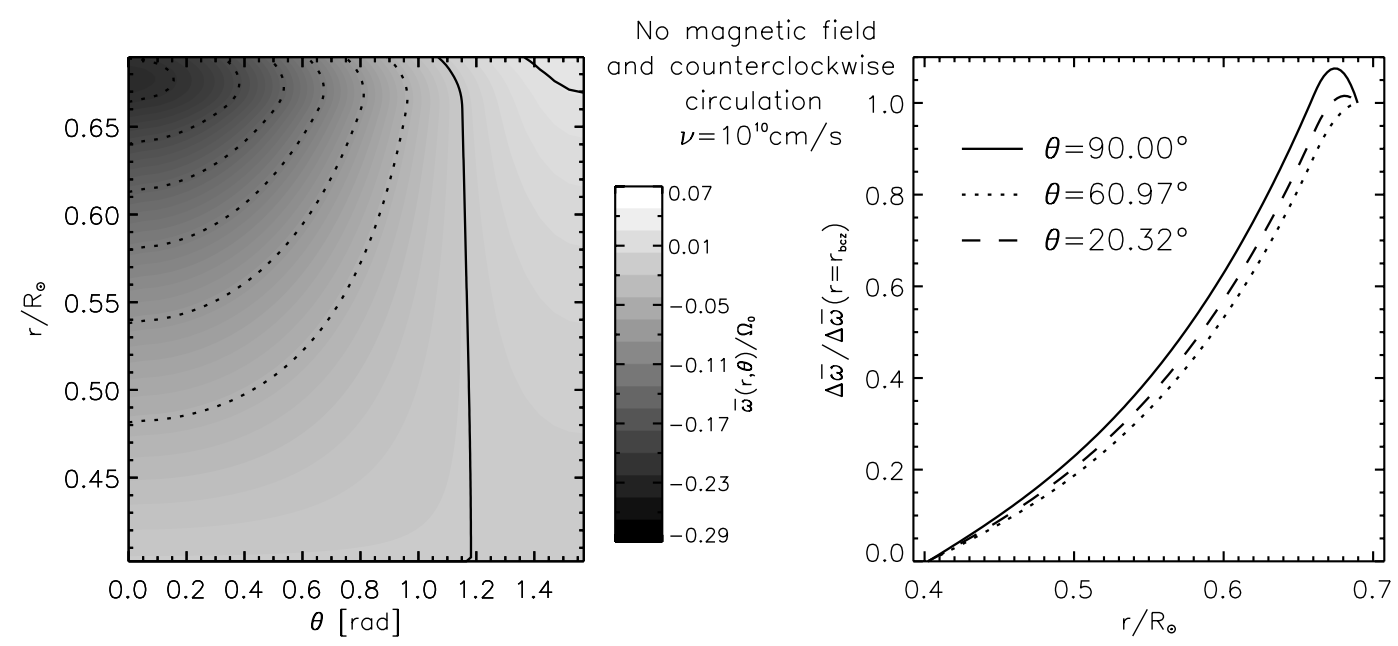

Fig. 13. Spreading of the differential rotation into the radiative interior including the meridional circulation shown in Fig. 9, but taking the imposed field to be null.
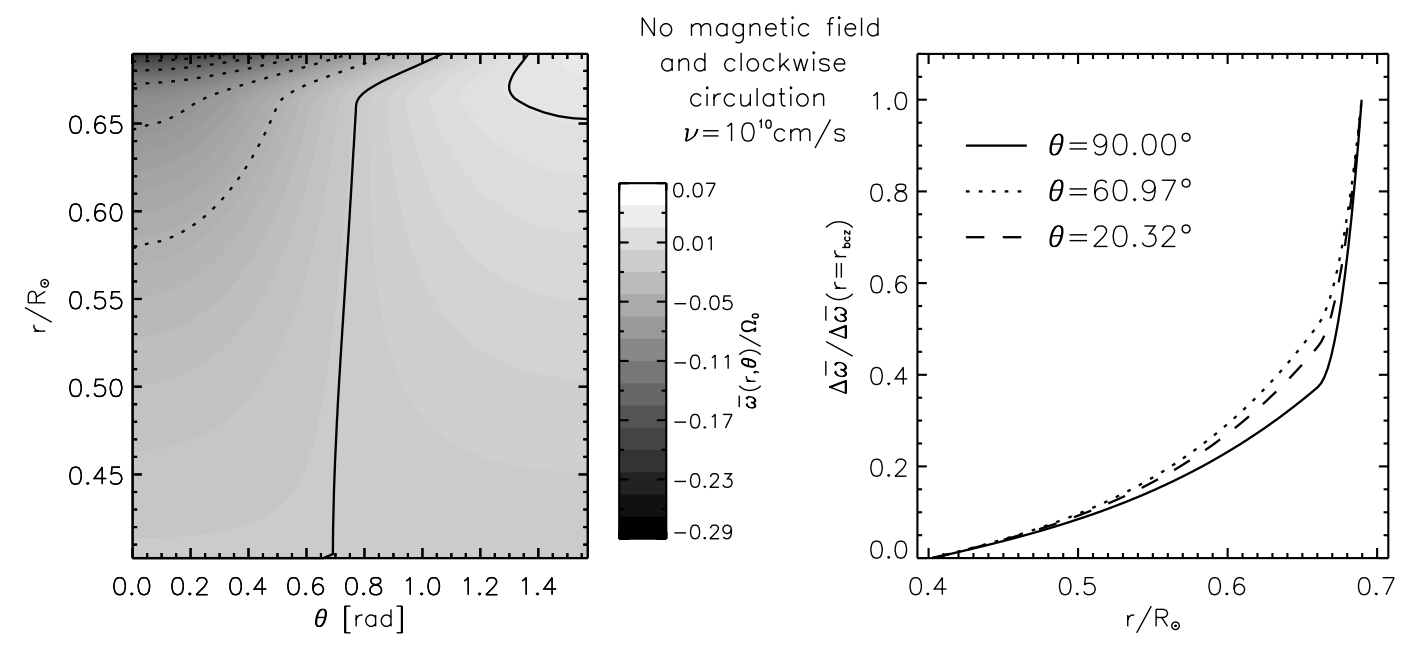

Fig. 14. Same as in Fig. 13 for the meridional circulation shown in Fig. 10.

equatorwards). Nevertheless, the resulting $B_{\text {conf }}$ values remain quite reasonable, in the range $10^{3}-10^{4} \mathrm{G}$, for all parameter combinations considered here.

The detailed dynamics of the solutions indicates that the thickness of the tachocline, in general, increases with latitude, and it also shows a rather strong dependence on cycle phase (Figs. 5 and 6 ). The latitude dependence is similar to that inferred by helioseismology, while the time dependence is within the observational errors.

The models with no meridional circulation also show a tendency for the rotational modulation to drift polewards (cf. Fig. 5). It must be noted, however, that owing to the rather simplified geometry of the poloidal magnetic field imposed on the upper boundary (oscillating dipole), the relevance of our results to the real Sun is dubious, as far as the detailed spatiotemporal structure of the tachocline is concerned. Another example of such features is the polar "pit" of slow rotation in nearly all of our models: again, the reality of this pit needs to be verified with other poloidal field geometries. A next step in our study of the fast tachocline should clearly be an extension of this investigation to more general magnetic field structures, reminiscent of the butterfly diagram.

Another important shortfall of these models is obviously the extremely simplified treatment of the turbulent transport, by constant scalar diffusivities. The turbulence present in the tachocline is presumably the product of complex MHD instabilities. The study of the nonlinear development of these instabilities has just begun (Cally 2001; Miesch 2001), and we are clearly a long way from being able to give a consistent description of tachocline turbulence. Nevertheless, the present models could still be significantly improved by considering simple, generic cases such as a scalar diffusivity coupled to the value of the local shear. Extensions of the present work along these lines will be treated in upcoming papers of this series.

Acknowledgements. The authors wish to thank the referee, Pascale Garaud, for helpful suggestions. We are also grateful to Sarbani Basu for useful insights on how to interpret 
helioseismic constraints on the tachocline, and to D. B. Guenther for making his solar model available. This work was supported in part by the National Science Foundation under grant no. PHY99-07949 and by the OTKA under grant no. T032462.

\section{References}

Basu, S., \& Antia, H. M. 2001, MNRAS, 324, 498

Brun, A. S. 2001, in Helio- and Asteroseismology at the Dawn of the Millennium (ESA Publ. SP-464), 273

Cally, P. S. 2001, Sol. Phys., 199, 231

Charbonneau, P., Christensen-Dalsgaard, J., Henning, R., et al. 1999, ApJ, 527, 445

Charbonneau, P., \& MacGregor, K. B. 1997, ApJ, 486, 502

Corbard, T., Berthomieu, G., Provost, J., \& Morel, P. 1998, A\&A, 330, 1149

Corbard, T., Blanc-Fraud, L., Berthomieu, G., \& Provost, J. 1999, A\&A, 344, 696
Corbard, T., Jiménez-Reyes, S. J., Tomczyk, S., Dikpati, M., \& Gilman, P. 2001, in Helio- and Asteroseismology at the Dawn of the Millennium (ESA Publ. SP-464), 265-272

Forgács-Dajka, E., \& Petrovay, K. 2001, Sol. Phys., 203, 195 (FDP01)

Garaud, P. 2001a, MNRAS, 324, 68

Garaud, P. 2001b, MNRAS, 329, 1

Gilman, P. A. 2000, Sol. Phys., 192, 27

Gilman, P. A., \& Dikpati, M. 2000, ApJ, 528, 552

Gough, D. O., \& McIntyre, M. E. 1998, Nature, 394, 755

Guenther, D. B., Demarque, P., Kim, Y.-C., \& Pinsonneault, M. H. 1992, ApJ, 387, 372

Komm, R. W., Howard, R. F., \& Harvey, J. W. 1993, Sol. Phys., 147, 207

Küker, M., \& Stix, M. 2001, A\&A, 366, 668

Latushko, S. 1994, Sol. Phys., 149, 231

MacGregor, K. B., \& Charbonneau, P. 1999, ApJ, 519, 911

Miesch, M. S. 2001, ApJ, 562, 1058

Rüdiger, G., \& Kitchatinov, L. L. 1997, Astr. Nachr., 318, 273 